\title{
Efficacy of prophylactic IV antibiotics in odontogenic bacteraemia prevention
}

\author{
Intravenous antibiotic regimens and prophylaxis of odontogenic bacteraemia
}

\section{G. Roberts and H. Holzel Br Dent J 2002; 193: 525-527}

\section{Objectives}

This study retrospectively examines the efficacy of prophylactic intravenous antibiotic regimens in the prevention of odontogenic bacteraemia in children with severe congenital heart defects receiving comprehensive dental treatment under general anaesthesia.

\section{Patients and methods}

Blood cultures were taken from children with congenital cardiac defects 30 seconds after completion of dental treatment under general anaesthesia. Antibiotic prophylaxis had been given intravenously immediately before dental treatment. The choice of antibiotics and the extent of dental treatment were recorded. The percentage prevalence of bacteraemia was compared with published data following multiple dental extractions using the same clinical and microbiological methodology.

Results

The overall percentage prevalence of positive cultures in children receiving intravenous prophylactic antibiotics was $16 \%$. The percentage of positive blood cultures in cardiac children who received Ampicillin alone was not significantly different from that in children having a combination of amikacin and teicoplanin $(16.7 \% \mathrm{v}$ $22.2 \%$ ) respectively [Chi Square $=0.385, \mathrm{df}=1, \mathrm{p}=0.535$ ]. When compared with multiple extractions, both ampicillin alone and amikacin with teicoplanin were effective in reducing the prevalence of odontogenic bacteraemia.

\section{Conclusions}

In children with cardiac defects, bacteraemia after dental treatment is reduced by antibiotics but is still detected on 16\% of occasions. In comparison with children receiving ampicillin alone, the combination of amikacin and teicoplanin is as effective in reducing bacteraemia in children who are either allergic to penicillins or who have received them within the previous month.

\section{IN BRIEF}

- Intravenous ampicillin is an effective drug regimen for reducing dental bacteraemia for comprehensive treatment under GA.

- If antibiotic resistant Staphylococcus aureus is a concern, alternative antibiotics to those published in the guidelines are required.

- A combination teicoplanin IV and amikacin IV proved to be as effective an antibiotic regimen as ampicillin IV.

\section{COMMENT}

The efficacy of prophylactic antibiotics in the prevention of infective endocarditis is still not proven. The ultimate experiment would involve two groups of patients at risk of infective endocarditis who were to have dental procedures done on them, which are known to be associated with bacteraemias. One group would receive prophylactic antibiotics the other would not. Unfortunately no ethical committee would approve such an experiment, as the consequences could be fatal for some of the patients not receiving antibiotics. This paper reports an indirect approach to assessing the efficacy of prophylactic antibiotic effectiveness by measuring the incidence of bacteraemias. The investigation by Roberts and Holzel has measured the incidence of bacteraemias following dental treatment in children with severe cardiac defects, who had been given prophylactic intravenous antibiotics. The number of children studied was impressive as this type of patient is thankfully rare, but 92 were included in the trial.

A wide range of intravenous antibiotics were used including amikacin, an agent that is not recommended by the Infective Endocarditis Working Party of the British Society for Antimicrobial Chemotherapy. Intravenous ampicillin was often used in this study in preference to amoxicillin, presumably on grounds of cost; this is a fairly widespread practice. Intravenous antibiotics did not eradicate bacteraemias and the incidence of positive blood cultures was $16 \%$ in the children studied. Some of the intravenous antibiotics were used on small numbers of patients and therefore meaningful conclusions could not be made. Ampicillin was found to be effective in reducing bacteraemias. A combination of intravenous amikacin and teicoplanin was found also to be effective in reducing bacteraemias in patients allergic to penicillin or who had received the antibiotic in the last month.

This paper provides objective data on the effect of intravenous antibiotics on the incidence of bacteraemias in patients with severe cardiac defects. None of these children developed infective endocarditis so this again provides indirect evidence that intravenous antibiotic prophylaxis does work. Sadly, extensive dental treatment was necessary in the group of children studied. The authors rightly point out that prevention of dental disease in the children would be far preferable to providing prophylactic intravenous antibiotics used as an adjunct treatment of their poor dentitions.

Dr M. V. Martin, Senior Lecturer/ Honorary Consultant Microbiologist, Department of Clinical Dental Science, University of Liverpool 